\title{
Relação entre atividade física e desportiva, níveis de IMC, perce- ções de sucesso e rendimento escolar
}

\author{
Relation between sport and physical activity, BMI levels, perceptions of suc- \\ cess and academic performance
}

Fernando Bastos ${ }^{1}$, Victor Machado Reis ${ }^{2,3}$, Ágata Cristina Aranha ${ }^{2,3}$, Nuno Domingos Garrido $^{2,3 *}$

\begin{abstract}
O excesso de peso é cada vez maior entre da população escolar jovem. Uma vez que não é evidente qual a atitude dos alunos com sobrepeso e obesidade face à participação em atividades físicas e sua relação com o desempenho escolar pretendemos identificar os factores de influência no contexto da EF escolar. Participaram neste estudo 490 alunos dos $3^{\circ}$ ciclo e ensino secundário, divididos por sexo. Os valores de IMC foram ajustados para sexo e idade e criados três grupos: Magreza; peso normal; e excesso de peso. Os valores dos resultados escolares foram normalizadas para uma escala de 1 a 5 valores. A participação em atividades desportivas, regularidade de prática e percepção do sucesso escolar foi medida por questionário. Após análise exploratória dos dados foram realizados os testes do Qui-quadrado, ANOVA e correlação de Pearson. O eta quadrado parcial $\left(\eta^{2}\right)$ foi reportado como medida da magnitude do efeito das diferenças verificadas nas ANOVAs. Foi considerado o valor de $\alpha$ de 0.05 . Os resultados mostraram que alunos com excesso de peso e magreza reportaram menores níveis de atividade física em relação a alunos normoponderais $\left(4.20 \pm 1.73,4.06 \pm 1.77,4.61 \pm 1.73 ; p=0.044 ; \eta^{2}=0.01\right.$, resp.). A maior parte dos alunos definiu o seu sucesso escolar através da aprovação/transição de ano a par de boas classificações, tendo este grupo obtido níveis superiores de prática (4.57 $\pm 1.71, \mathrm{p}=0.03)$ Verificaram-se efeitos significativos da variável níveis de IMC nas classificações médias finais e na disciplina de EF, em que os alunos normoponderais obtiveram melhores classificações do que os alunos com excesso de peso. Além disso, verificaram-se correlações significativas entre as classificações de Português e Matemática $(r=0.80 ; p<0.001)$, entre as classificações de EF e Português $(r=0.36 ; p<0.001)$, e entre as classificações de EF e Matemática $(r=0.42 ; p<0.001)$. Os alunos com sobrepeso apresentaram níveis inferiores de prática de atividade física quando comparados com os alunos com peso normal ou magros concomitantemente com um rendimento escolar inferior. Face aos resultados consideramos a prática de atividade física um fator com influência positiva no rendimento escolar em alunos dos ensinos básico e secundário.
\end{abstract}

Palavras-chave: Atividade física, IMC, perceções de sucesso, rendimento escolar, EF.

ABSTRACT

Overweight is increasing among the school population being physical inactivity one of its main causes. Since it is not clear what kind of attitude do overweight and obese students have towards participation in physical activity and its relationship with academic performance we intend to identify the influencing factors in the context of physical education. Participated in this study 490 students from $3^{\text {rd }}$ cycle and secondary education, divided by gender. BMI values were adjusted for sex and age and three groups were created: leanness; normal weight; and overweight. The values of the academic performance were normalized to a scale of 1 to 5 points. Participation in sporting activities, regularity of practice and perception of academic performance were measured by questionnaire. After exploratory data analysis, chi-square test, ANOVA and Pearson correlation were performed. The partial eta squared $\left(\eta^{2}\right)$ was reported as a measure of the magnitude of the effect of the differences in the ANOVAs. We considered the value of $\alpha$ of 0.05 . The results showed that students with overweight and thinness reported lower levels of PA compared to normal weight students $\left(4.20 \pm 1.73,1.77 \pm 4.6,4.61 \pm 1.73, \mathrm{p}=0.044, \eta^{2}=0: 01\right.$, resp.). Most students defined its academic success through the approval /transition from year along with good classifications, having this group obtained higher levels of PA $(4.57 \pm 1.71, \mathrm{p}=0.03)$. There were significant effects of BMI levels in the final average and Physical Education ratings in which normal weight students had better ratings than overweight counterparts. Furthermore, there were significant correlations between the ratings of Portuguese and Mathematics $(r=0.80, p<0.001)$, between classifications of Physical Education and Portuguese $(r=0: 36, p<0.001)$, and between classifications of Physical Education and Mathematics $(r=0: 42, p<0.001)$. Overweight students had lower levels of PA when compared to students with normal or lean weight concomitantly with lower school performance levels. Given the results we consider that practicing PA is a factor with a positive influence on the academic performance of students in basic and secondary education.

Keywords: physical activity, BMI, perceptions of success, academic performance, physical education

\footnotetext{
Artigo recebido a 17.04.2014; Aceite a 22.05.2015

${ }^{1}$ Instituto Federal de Belém do Pará, Belém do Pará, Brasil

${ }^{2}$ Universidade de Trás-os-Montes e Alto Douro, DCDES, Vila Real, Portugal

${ }^{3}$ Centro de Investigação em Desporto, Saúde e Desenvolvimento Humano (CIDESD), Portugal

* Autor correspondente: UTAD - Quinta de prados, Complexo desportivo, 5000-000 Vila Real, Portugal, E-mail: ndgarrido@gmail.com
} 


\section{INTRODUÇÃO}

Nos países da União Europeia o sobrepeso e obesidade estão fortemente associados com estilos de vida sedentários e com a ausência de atividade física (AF) na população adulta (MartínezGonzález, Martínez, Hu, Gibney, \& Kearney, 1999) sendo a inatividade física um fator determinante na acumulação excessiva de gordura (McArdle, Katch, \& Katch, 2010). Como agravante, o estilo de vida contemporâneo e modernização convidam também a população mais jovem a práticas de sedentarismo e aumento da prevalência de obesidade (Ogden et al., 2006). É sabido que a AF regular está associada com melhorias no bem-estar e saúde, assim como das capacidades físicas dos praticantes (Blair, LaMonte, \& Nichaman, 2004) onde os benefícios para a saúde parecem ser proporcionais à quantidade de AF realizada (US Department of Health and Human Services, 1996). Se por um lado a relação da AF com a saúde está refletida no efeito protetor da mesma reduzindo do risco de aparecimento de doenças degenerativas e controláveis (Eckel, Grundy, \& Zimmet, 2005) por outro é de esperar que a AF também atrase a mortalidade e aumente a longevidade (Lee, Paffenbarger, \& Hennekens, 1997). De facto, os fatores de risco nos adultos podem ter a sua origem na infância ou adolescência e podem ser atenuados e/ou evitados através da AF regular nessas idades (Freedman, Khan, Dietz, Srinivasan, \& Berenson, 2001). É então fundamental aumentar a consciência e a participação de crianças e adolescentes em programas regulares e estruturados de prática (Mota \& Sallis, 2002), pois estas faixas etárias representam o período ótimo para estimular os hábitos de um estilo de vida saudável que, se adquiridos, provavelmente manter-se-ão durante a idade adulta (Shephard \& Trudeau, 2000).

A educação física (EF) e a escola estão na linha da frente e são considerados contextos de intervenção fulcrais para o desenvolvimento de estilos de vida ativos nos mais novos (WHO/CDC, 2005), apesar da aparente menor importância dada à EF relativamente a outras áreas disciplinares (Kelder et al., 2003). Contudo, programas aplicados com base em evidências que comprovam benefícios, quer na saúde, quer no sucesso escolar fazem da EF um importante recurso a ter em conta (Sallis et al., 2012). Ao ser o primeiro contexto com responsabilidade de promover a AF em crianças e jovens (Sallis \& Owen, 1998), a EF escolar pode ser o caminho no combate à inatividade física e hábitos sedentários, uma vez que as crianças estão cada vez menos ativas (Armstrong, 1995; Armstrong \& Van Mechelen, 1998) e a sua participação em atividades físicas tem diminuído com o aumento da idade (Armstrong \& Welsman, 2006).

Além dos benefícios apontados também há a sugestão de que o tempo dedicado à EF ou a outras atividades físicas não tem impacto negativo no sucesso escolar e pode, inclusive, estar ligado à obtenção de melhores notas (Rasberry et al., 2011), contudo esta evidência, apesar de existir, ainda não é totalmente considerada para o efeito (Donnelly et al., 2013).

Uma vez que ainda não está claro nem evidente qual a atitude dos alunos com sobrepeso e obesidade face à participação em atividades físicas e desportivas e sua relação com o desempenho escolar definimos como objetivo central deste estudo a identificação dos fatores de influência no contexto da EF escolar. Desta forma, os objetivos específicos deste estudo foram caracterizar os níveis de prática de $\mathrm{AF}$ e desporto federado; caracterizar os níveis de IMC; verificar quais as perceções de sucesso escolar e fatores que o influenciam; e caracterizar o rendimento escolar e verificar os fatores que o influenciam.

\section{MÉTODO}

A presente investigação é de natureza quantitativa, de acordo com um delineamento quasi-experimental, descritivo e correlacional, tendo os dados sido recolhidos num único momento temporal (transversal).

\section{Amostra}

A amostra do presente estudo foi constituída por um total de 490 alunos dos ensinos básico e secundário, com idades compreendidas entre os 12 e os 18 anos. Relativamente ao sexo, 245 (50.0\%) eram do sexo masculino e 245 (50.0\%) eram do sexo feminino. No que concerne a idade, os alunos foram agrupados em duas faixas etárias: $12-15$ anos $(\mathrm{n}=155 ; 31.6 \%)$ e $16-18$ anos $(n=335 ; 68.4 \%)$. Em termos de ciclo de ensino, 
325 (66.3\%) estavam matriculados num ano de escolaridade do $3^{\circ}$ ciclo, enquanto os restantes $165(33.7 \%)$ estavam matriculados no $10^{\circ}, 11^{\circ}$ ou $12^{\circ}$ ano (ensino secundário). Os valores médios do IMC variaram entre 12.99 e $38.48 \mathrm{~kg} / \mathrm{m}^{2}$ (20.54 \pm 3.48$)$.

Os alunos foram recrutados em vários estabelecimentos de ensino da região litoral de Portugal, segundo um processo de amostragem nãoprobabilístico (intencional). Procedeu-se à obtenção da autorização formal dos diretores das escolas e dos pais/encarregados de educação para a aplicação dos questionários, garantindo o anonimato e confidencialidade dos dados recolhidos. O presente estudo foi aprovado pelo comité de ética institucional e respeitou as diretrizes internacionais sobre ética em pesquisas científicas.

\section{Instrumentos}

A recolha de dados foi efetuada em contexto de sala de aula, através da utilização de computadores com ligação à internet, tendo os alunos preenchido os dados num formulário online criado e disponível unicamente para este efeito. Este processo decorreu sob a presença do primeiro investigador de modo a uniformizar os procedimentos de administração do questionário e dar instruções precisas aos alunos, quando solicitado, de forma a minimizar a possível ocorrência de erros interpretativos. O preenchimento dos dados referentes à massa corporal e estatura foi feito pelos alunos no mesmo formulário, tendo por referência os valores obtidos numa medição efetuada na aula anterior de EF, de acordo com o manual do Fitnessgram (NES, 2002). A massa corporal foi medida através de balança eletrónica, com a possibilidade de obter valores aproximados aos 100 gramas. A massa corporal foi registada com o aluno descalço, com roupas leves, na posição antropométrica e com os pés apoiados no centro da balança. O registo foi feito em quilogramas com um valor decimal. A estatura foi medida com recurso a um estadiómetro portátil, após uma inspiração profunda. As medições foram realizadas na posição antropométrica, com a cabeça posicionada segundo o plano de Frankfurt. O registo de dados foi efetuado em centímetros e posteriormente convertido para metros. As classificações obtidas às várias disciplinas no período lectivo anterior foram igualmente preenchidas pelos alunos, segundo a ficha individual de informação escolar que estes foram solicitados a trazer no dia do preenchimento do questionário. Tendo em consideração as diferentes escalas de classificação dos ensinos básico e secundário, procedeu-se posteriormente à normalização das classificações dos alunos do ensino secundário numa escala de 5 valores.

A categorização dos adolescentes por níveis de IMC foi efetuada segundo as propostas de Cole e colaboradores (eg. Cole, Bellizzi, Flegal, \& Dietz, 2000; Cole, Flegal, Nicholls, \& Jackson, 2007; Cole \& Lobstein, 2012), ajustadas para o sexo e idade. Deste modo, foram criados três grupos: magreza, peso normal e excesso de peso. O grupo com excesso de peso incluiu simultaneamente os adolescentes com pré-obesidade e obesidade.

A tradução e adaptação transcultural dos instrumentos utilizados seguiram um conjunto de processos de tradução e retroversão efetuados por diferentes indivíduos bilingues, não familiarizados com os instrumentos. A adequação semântica e validade de conteúdo foram estabelecidas através de um painel de especialistas, com habilitações e experiência na área científica em causa, tendo-se obtido uma versão final, traduzida e adaptada, que reuniu consenso por parte dos membros do painel.

\section{Procedimentos}

Inicialmente, os alunos responderam um conjunto de questões de natureza sociodemográfica (sexo, idade, ano de escolaridade e agregado familiar), assim como foram solicitadas as medições de massa corporal e estatura, e as classificações escolares.

As questões do nosso estudo sobre a mensuração da atividade física-desportiva, aplicadas noutros estudos (Cloes, 2004; Cloes, Laraki, Dubuisson, \& Theunissen, 2007), foram baseados no modelo desenvolvido por Prochaska, Sallis e Long (2001), onde a frequência de prática de AF foi mensurada através da média de dois itens ("Ao longo dos últimos 7 dias, quantas vezes andaste a pé ou de bicicleta durante pelo menos 10 minutos, para ir para a escola, ir ter com amigos, 
ir às compras ou apenas para andar?" e "Ao longo dos últimos 7 dias, quantas vezes fizeste uma atividade física durante pelo menos 20 minutos, e que te fez acelerar a respiração ou te fez suar"), enquanto a prática desportiva federada foi avaliada através de um item ("Ao longo dos últimos 7 dias, quantas horas praticaste fora da escola, ou em uma estrutura organizada/federada"). A regularidade da prática de $\mathrm{AF}$ e/ou desporto federado foi mensurada através da questão "Praticas um desporto (ou atividade física) fora da escola...", com as seguintes opções: sim, durante todo o ano; sim, mas não durante todo o ano; Não.

As perceções do sucesso escolar dos alunos basearam-se na resposta à questão "Para ti, o sucesso escolar é...", com as seguintes opções: passar de ano, mesmo com negativas; passar de ano sem qualquer negativa; passar de ano com boas notas.

\section{Análise estatística}

Primeiramente recorremos aos procedimentos habituais da estatística descritiva (média, desvio-padrão e frequência relativa) e análise da normalidade univariada dos dados (simetria e achatamento). Para efeitos de análise inferencial utilizámos os testes do Qui-quadrado, ANOVA uni e multivariada e correlação de Pearson. O eta quadrado parcial $\left(\eta^{2}\right)$ foi reportado como medida da magnitude do efeito das diferenças verificadas nas ANOVAs, utilizando-se os seguintes critérios de interpretação: $>0.01$ (efeito pequeno), $>0.06$ (efeito moderado) e $>0.14$ (efeito elevado) (Cohen, 1988). As propriedades psicométricas das dimensões do questionário foram investigadas através da análise fatorial confirmatória e alfa de Cronbach, tendo revelado um bom índice de consistência interna (alfa de Cronbach igual a $0,73)$ e razoável adequação fatorial (CFI> 0,90 e RMSEA <0,08). O nível de significância foi mantido em 5\% ( $\mathrm{p}<0.05)$. Os pacotes estatísticos utilizados foram o IBM SPSS Statistics 19.0 e o EQS 6.1

\section{RESULTADOS}

Os alunos, em média, praticavam AF em 4.49 \pm 1.74 dias da semana, sendo observável maior nível de prática por parte dos meninos $(4.73 \pm$
1.73) quando comparados com as meninas (4.24 $\pm 1.70 ; p=0.002 ; \eta^{2}=0.02$ ). A comparação por grupos etários revelou um aumento dos níveis de prática entre os $12-15$ anos $(4.02 \pm 1.74)$ e os 16 18 anos $\left(4.70 \pm 1.69 ; p<0.001 ; \eta^{2}=0.03\right)$. Por sua vez, alunos com excesso de peso (4.20 \pm $1.73)$ e magreza $(4.06 \pm 1.77)$ reportaram menores níveis de AF em relação a alunos normoponderais $\left(4.61 \pm 1.73 ; \mathrm{p}=0.044 ; \eta^{2}=0.01\right)$.

No que concerne à regularidade da prática, $320(65.3 \%)$ afirmaram fazê-la de forma regular, $106(21.6 \%)$ reportaram praticar de modo irregular (sazonal ou ocasionalmente) e 64 (13.1\%) indicaram não serem praticantes. Estas prevalências apresentaram diferenças por sexo $(p=0.01)$, verificando-se uma maior prevalência de prática regular nos meninos $(71.8 \%$ vs. $58.8 \%)$ e maiores prevalências de prática irregular ou não prática nas meninas $(25.7 \%$ vs. $17.6 \%$ e $15.5 \%$ vs. $10.6 \%$, respetivamente). O grupo etário, níveis de IMC e agregado familiar não influenciaram significativamente a regularidade da prática $(\mathrm{p}>$ 0.05).

Relativamente à prática desportiva federada, a amostra revelou praticar cerca de 3.21 horas (DP $=2.59$ ) por semana, tendo-se obtido níveis superiores nos meninos $(3.73 \pm 2.56$ vs. $2.69 \pm 2.52$; $\left.p<0.001 ; \eta^{2}=0.04\right)$. No entanto, as variáveis grupo etário e níveis de IMC não produziram efeitos significativos nos níveis de envolvimento desportivo federado ( $\mathrm{p}>0.05)$. No que concerne a regularidade da prática desportiva federada, 288 (58.8\%) afirmaram fazê-la de forma regular, 78 (15.9\%) reportaram praticar de modo irregular (sazonal ou ocasionalmente) e 124 (25.3\%) indicaram não serem praticantes federados. Estas prevalências apresentaram diferenças por sexo ( $\mathrm{p}$ $<0.001$ ), verificando-se uma maior prevalência de prática regular nos meninos $(69.4 \%$ vs. $48.2 \%)$ e maiores prevalências de prática irregular ou não prática nas meninas ( $18.8 \%$ vs. $13.1 \%$ e $33.1 \%$ vs. $17.6 \%$, respetivamente). O grupo etário e níveis de IMC não influenciaram significativamente a regularidade da prática desportiva federada ( $\mathrm{p}>0.05)$.

Os alunos da amostra revelaram níveis médios de IMC de $20.54 \pm 3.48 \mathrm{~kg} / \mathrm{m}^{2}$, variando entre 12.99 e $38.48 \mathrm{~kg} / \mathrm{m}^{2}$. As análises comparativas do 
IMC por sexo não indicaram diferenças significativas ( $p>0.05$ ). No entanto, e como esperado, verificou-se que alunos mais velhos evidenciaram níveis médios mais elevados $(20.97 \pm 3.27)$ em comparação com os alunos mais novos (19.61 \pm 3.75; $\left.\mathrm{p}<0.001 ; \eta^{2}=0.03\right)$. Por sua vez, a análise por níveis de IMC (magreza, peso normal e excesso de peso) indicou 3.3\% ( $\mathrm{n}=16)$ com magreza, $71.4 \%(\mathrm{n}=350)$ com peso normal e $25.3 \%$ $(\mathrm{n}=124)$ com excesso de peso. As prevalências de excesso de peso e magreza foram superiores no sexo masculino em comparação ao sexo feminino $(27.8 \%$ vs. $22.9 \%$ e $5.3 \%$ vs. $1.2 \%$, respetivamente), enquanto se observou uma maior percentagem de meninas no grupo normoponderal (75.9\% vs. $66.9 \%$; $\mathrm{p}=0.01$ ).

Destaca-se que grande parte dos alunos define o seu sucesso escolar através da aprovação/transição de ano a par de boas classificações (tabela 1). Verificaram-se efeitos significativos do sexo e níveis de IMC, no qual se observou uma maior prevalência de meninas e adolescentes com peso normal no grupo das perceções associadas à aprovação/transição de ano com boas classificações. Por sua vez, denotou-se uma maior percentagem de meninos e adolescentes com magreza no grupo dos que dependiam de obter aprovação/transição de ano, mesmo que tal implicasse ter classificações negativas. Relativamente à comparação dos níveis de AF por grupos de perceções de sucesso escolar, somente se identificou uma diferença significativa $(p=0.03)$, em que os alunos que definiam o seu sucesso escolar através da aprovação/transição de ano com boas classificações reportaram níveis superiores de prática $(4.57 \pm 1.71)$ quando comparados com os alunos para os quais bastaria obter aprovação/transição de ano sem classificações negativas (3.99 \pm 1.73). Por sua vez, não se verificaram diferenças significativas de prática desportiva federada em função de diferentes grupos de perceções de sucesso escolar $(p>0.05)$.

Tabela 1

Associação entre perceçôes de sucesso escolar por sexo, grupo etário e níveis de IMC

\begin{tabular}{lcccc}
\hline \multicolumn{1}{c}{ Variáveis } & Passar de ano com & Passar de ano sem & Passar de ano com & $p$ \\
\hline Sexo & & & & \\
Masculino & $6.5 \%$ & $17.6 \%$ & $75.9 \%$ & 0.01 \\
Feminino & $1.6 \%$ & $10.6 \%$ & $87.8 \%$ & \\
Grupo etário & & & & \\
12-15 anos & $2.6 \%$ & $12.9 \%$ & $84.5 \%$ & 0.43 \\
16-18 anos & $4.8 \%$ & $14.6 \%$ & $80.6 \%$ & \\
Níveis de IMC & $18.8 \%$ & $18.8 \%$ & $62.4 \%$ & 0.01 \\
Magreza & $3.1 \%$ & $12.3 \%$ & $84.6 \%$ & $76.6 \%$ \\
Peso normal & $4.8 \%$ & $18.5 \%$ & & \\
Excesso de peso & & & & \\
\hline
\end{tabular}

Os resultados do rendimento escolar indicaram os seguintes valores: média das classificações (3.72 \pm 0.71$)$, classificações de Português (3.53 $\pm 0.86)$, Matemática $(3.45 \pm 1.02)$ e EF $(4.22 \pm$ 0.63). Verificaram-se correlações significativas entre as classificações de Português e Matemática $(r=0.80 ; p<0.001)$, entre as classificações de EF e Português ( $r=0.36$; $p<0.001)$, e entre as classificações de EF e Matemática $(r=0.42$; $\mathrm{p}<$ 0.001).

A comparação em função do sexo indicou que as meninas obtiveram melhores classificações médias finais e nas disciplinas de Português e Matemática. Não se verificaram diferenças por sexo nas classificações de EF ( $p=0.11)$. A análise comparativa por grupos etários indicou melhores classificações médias finais e nas disciplinas de Português e Matemática por parte dos alunos com 12-15 anos, enquanto os alunos mais velhos (16-18 anos) obtiveram melhores classificações na disciplina de EF. Verificaram-se efeitos significativos da variável níveis de IMC nas classificações médias finais e na disciplina de EF, em que os alunos normoponderais obtiveram melhores classificações do que os alunos com excesso de peso.

A comparação em função das perceções de sucesso escolar revelou que o grupo das perceções 
associadas à aprovação/transição de ano com boas classificações obteve melhores classificações na média final e nas restantes disciplinas analisadas do que os restantes grupos.

Além disso, verificaram-se correlações significativas entre as classificações de Português e Matemática ( $r=0.80 ; p<0.001)$, entre as classificações de EF e Português $(r=0.36$; $p<0.001)$, e entre as classificações de EF e Matemática $(\mathrm{r}=$ $0.42 ; \mathrm{p}<0.001$ ).

A correlação entre as variáveis do rendimento escolar e a frequência de prática de AF unicamente indicou uma relação positiva significativa entre as classificações de EF e a prática AF ( $\mathrm{r}=$
$0.25 ; \mathrm{p}<0.001)$. Por sua vez, a frequência de prática desportiva federada relacionou-se positiva e significativamente com a classificação média final $(\mathrm{r}=0.11 ; \mathrm{p}<0.05)$ e as classificações de EF $(\mathrm{r}$ $=0.28 ; \mathrm{p}<0.001)$. Tendo em consideração a diferença de prática por sexo, efetuámos esta mesma análise separadamente por sexo masculino e feminino. Os resultados obtidos indicaram, além das evidências anteriormente enunciadas, uma relação significativa entre a prática desportiva federada por parte das meninas e as classificações na disciplina de Português $(r=0.17$; $\mathrm{p}<$ 0.05).

Tabela 2

Comparação do rendimento escolar em função do sexo, grupo etário, níveis de IMC, prática de AF e perceções de sucesso escolar

\begin{tabular}{|c|c|c|c|c|c|c|c|c|}
\hline Variáveis & $\begin{array}{l}\text { Média final } \\
\mathrm{M} \pm \mathrm{DP}\end{array}$ & $\eta 2$ & $\begin{array}{c}\text { Português } \\
\mathrm{M} \pm \mathrm{DP}\end{array}$ & $\eta 2$ & $\begin{array}{c}\text { Matemática } \\
\mathrm{M} \pm \mathrm{DP}\end{array}$ & $\eta 2$ & $\begin{array}{c}\mathrm{EF} \\
\mathrm{M} \pm \mathrm{DP} \\
\end{array}$ & $\eta 2$ \\
\hline \multicolumn{9}{|l|}{ Sexo } \\
\hline Masculino & $3.61 \pm 0.67$ & \multirow{2}{*}{0.04} & $3.38 \pm 0.82$ & \multirow{2}{*}{0.04} & $3.33 \pm 0.99$ & \multirow{2}{*}{0.04} & $4.27 \pm 0.61$ & \\
\hline Feminino & $3.82 \pm 0.73^{*}$ & & $3.68 \pm 0.88^{*}$ & & $3.58 \pm 1.04^{*}$ & & $4.17 \pm 0.64$ & \\
\hline \multicolumn{9}{|l|}{ Grupo etário } \\
\hline $12-15$ anos & $3.94 \pm 0.74$ & & $3.83 \pm 0.91^{¥}$ & \multirow{2}{*}{0.11} & $3.75 \pm 1.04^{¥}$ & \multirow{2}{*}{0.11} & $4.11 \pm 0.76$ & \multirow{2}{*}{0.11} \\
\hline $16-18$ anos & $3.62 \pm 0.67$ & & $3.39 \pm 0.80$ & & $3.31 \pm 0.98$ & & $4.27 \pm 0.55^{*}$ & \\
\hline \multicolumn{9}{|l|}{ Níveis de IMC } \\
\hline Magreza & $3.45 \pm 0.71$ & \multirow{3}{*}{0.02} & $3.16 \pm 0.82$ & & $3.06 \pm 1.02$ & & $4.05 \pm 0.71$ & \multirow{3}{*}{0.09} \\
\hline Peso normal & $3.77 \pm 0.71^{*}$ & & $3.58 \pm 0.84$ & & $3.50 \pm 1.03$ & & $4.33 \pm 0.59^{*}$ & \\
\hline Excesso de peso & $3.58 \pm 0.69$ & & $3.39 \pm 0.92$ & & $3.34 \pm 0.99$ & & $3.90 \pm 0.62$ & \\
\hline \multicolumn{9}{|c|}{ Percepçôes de sucesso escolar } \\
\hline $\begin{array}{l}\text { Passar de ano com } \\
\text { classificações } \\
\text { negativas }\end{array}$ & $2.83 \pm 0.21$ & & $2.56 \pm 0.50$ & & $2.23 \pm 0.44$ & & $3.82 \pm 0.67$ & \\
\hline $\begin{array}{l}\text { Passar de ano sem } \\
\text { classificações } \\
\text { negativas }\end{array}$ & $3.29 \pm 0.56$ & 0.08 & $3.01 \pm 0,79$ & 0.08 & $2.98 \pm 0.80$ & 0.08 & $4.00 \pm 0.66$ & 0.08 \\
\hline $\begin{array}{l}\text { Passar de ano com } \\
\text { boas classificações }\end{array}$ & $3.82 \pm 0.69^{\#}$ & & $3.65 \pm 0.83^{\#}$ & & $3.58 \pm 1.02^{\#}$ & & $4.35 \pm 0.61^{\#}$ & \\
\hline
\end{tabular}

\section{DISCUSSÃO}

O objetivo deste estudo foi caracterizar os níveis de prática de AF e desporto federado; caracterizar os níveis de IMC; verificar quais as perceções de sucesso escolar e fatores que o influenciam; e caracterizar o rendimento escolar e verificar os fatores que o influenciam. Tanto quanto a literatura nos permite conhecer, o presente estudo constitui-se como inédito na análise da perceção do sucesso escolar dos alunos do ensino básico e secundário e a sua relação com o sucesso escolar, níveis de IMC e prevalência de prática de AF.

\section{Prática desportiva}

Os alunos do nosso estudo apresentaram uma média de $4.49 \pm 1.74$ dias por semana de prática destacando-se o maior nível de prática dos meninos em comparação com as meninas. Historicamente tem sido assim no panorama desportivo, o que eventualmente terá tido reflexos também na prática de AF. Mais recentemente os estudos científicos têm apresentado uma tendência convergente com este fenómeno (Armstrong \& Welsman, 2006; Maffeis \& Castellani, 2007; Yli-Piipari, Leskinen, Jaakkola, \& Liukkonen, 2012) e os nossos dados não são exceção. 
Do ponto de vista da prevalência, os meninos do nosso estudo tiveram uma prevalência de prática regular $13 \%$ superior à das meninas $(\mathrm{p}=$ 0.01 ). Estes resultados estão em linha com os do estudo de Sallis (1993), onde mostrou que meninos eram $20 \%$ mais ativos que as meninas e que a AF média diminuía $2.7 \%$ com a idade nos meninos e $7.4 \%$ nas meninas. Este último facto não foi confirmado no nosso estudo revelando resultados inversos. Outro exemplo é o estudo do U.S. National Youth Risk Behavior Survey, com mais de 11.000 adolescentes do ensino secundário, onde Heath, Pratt, Warren, e Kann (1994) verificaram que a percentagem de meninas adolescentes que eram ativas no $9^{\circ}$ ano diminuiu de $31 \%$ para $17 \%$ até ao $12^{\circ}$.

Os meninos podem apresentar maiores níveis de prática por vários motivos. Um deles que não foi abordado no questionário pode dever-se ao facto dos pais dos meninos terem maiores níveis de prática de $\mathrm{AF}$ que os pais das meninas. Relativamente a este tópico, Taylor, Baranowski, e Sallis (1994) reforçam o papel fundamental da família na participação desportiva, quer como modelos a seguir, quer como suporte e influência social, encontrando aceitação ainda hoje em literatura mais recente que indica que os meninos têm mais apoio social e familiar para a realização de atividades físicas (Gonçalves, Hallal, Amorim, Araújo, \& Menezes, 2007), onde o apoio e incentivo dos pais têm apresentado mais valor preditivo que o nível de prática de AF dos mesmos (Trost et al., 2003).

Encontramos também uma relação positiva entre a classificação a EF (médias acima dos 4 pontos), superior nos meninos, e a prática de $\mathrm{AF}$ $(\mathrm{r}=.25 ; \mathrm{p}<0.05)$. Contudo, se retirarmos a EF e incluirmos os dados da prática desportiva federada, verificamos um efeito do sexo nos níveis de prática $(\mathrm{p}<0.001)$ onde os meninos indicaram uma participação mais elevada que as meninas. Estas relações matemáticas, apesar de mostrarem a tendência não explicam a causa, que não é única. As normas e os fatores socioculturais assim como as expetativas da sociedade relativamente à participação desportiva influenciam a distinção entre os níveis de prática dos dois sexos (Weinberg \& Gould, 1997). Além da tendência das crianças responderem a estereótipos sociais em relação ao desporto e AF, outra explicação pode surgir do facto dos meninos tradicionalmente apresentarem maior competência motora que as meninas (Mateus, Dias, Corte-Real, Garganta, \& Fonseca, 2010). A competência motora parece ser necessária para assegurar algum sucesso durante a AF (Cliff, Okely, Smith, \& McKeen, 2009) estando associada à participação desportiva dos jovens (Jaakkola et al., 2009; Okely, Booth, \& Patterson, 2001).

Outro fator que pode explicar a menor prevalência de participação das meninas é a intensidade das atividades físicas. A nível dos países europeus, incluindo Portugal, os meninos apresentam níveis de AF superior às meninas, aumentando esta diferença quando se considera AF vigorosa (Armstrong \& Welsman, 2006). O elevado nível de competição e intensidade nos desportos federados pode levar a que alguns não participem (Landry \& Driscoll, 2012). Além de participarem menos em AF e atividades federadas, normalmente as meninas participam menos nas aulas de EF e a sua participação normalmente é em atividades com menor intensidade (McKenzie, Marshall, Sallis, \& Conway, 2000).

Não foi feito no nosso estudo uma descriminação das modalidades praticadas, uma vez que estava fora do âmbito do mesmo. Os jogos desportivos coletivos têm uma prevalência maior durante a adolescência (Azevedo Júnior, Araújo, \& Pereira, 2006), mas consideramos provável que as modalidades individuais possam ter mais aceitação entre as meninas e a sua participação ser realizada com mais confiança (Corbin, 2002).

A redução dos níveis de AF com o aumento da idade começa mais cedo nas meninas (Maffeis \& Castellani, 2007) e, apesar de não termos dados disponíveis para verificar na nossa amostra onde se iniciou essa redução, constatamos algo importante para caracterizar a menor prevalência de prática por parte das meninas. As meninas do nosso estudo demonstraram maiores níveis de inatividade física que os meninos $(p=0.01)$. Esta tendência está bem documentada por Oehlschlaeger, Pinheiro, Horta, Gelatti, e San'Tana (2004) referindo que a prevalência do sedentarismo é 2.45 maior em meninas que em meninos. 
No nosso caso é aproximadamente 1.5 vezes maior (15.5 vs 10.6\%). Independentemente da dimensão do valor, são dados preocupantes.

Armstrong e Welsman (2006) mostraram que o resultado da população portuguesa nos níveis de AF é preocupante. Nos meninos de 11 e 13 anos apenas $40 \%$ cumprem os níveis mínimos recomendados, sendo de aproximadamente 30\% nos meninos de 15 anos. Nas meninas, o cenário agrava-se ainda mais com valores de aproximadamente $22.5 \%$ nos 11 anos, $15 \%$ nos 13 anos e $10 \%$ nos 15 anos. Dos 22 países estudados, apenas 4 estão com níveis inferiores a Portugal.

Estudos realizados na população portuguesa (Guerra, Santos, Ribeiro, Duarte, \& Mota, 2003; Mota, Santos, Guerra, Ribeiro, \& Duarte, 2002; Santos, Guerra, Ribeiro, Duarte, \& Mota, 2003) mostraram resultados impressionantes, uma vez que $94 \%$ dos meninos e $79 \%$ das meninas acumularam pelo menos 60 minutos de AF de intensidade moderada a vigorosa por dia e que o tempo passado em AF aumentava com o aumento da idade. Estes estudos foram realizados com recurso a acelerómetros. O nosso estudo foi realizado através de métodos subjetivos por questionário. É necessário ter cautela a comparar os resultados uma vez que também são muito diferentes do reportado por Armstrong e Welsman (2006) que apresentaram dados obtidos por métodos subjetivos.

Um dado interessante que diverge da literatura é o aumento do nível de prática com o aumento da idade da faixa 12-15 para a faixa 16-18 $(4.02 \pm 1.74$ vs $4.70 \pm 1.69 ; \mathrm{p}<0.001)$. Estes resultados fogem da tendência da generalidade dos dados publicados relativamente aos níveis de prática. Alguns estudos reportaram aumentos dos níveis de AF com o aumento da idade (Guerra et al., 2003; Mota et al., 2002; Santos et al., 2003; Vasconcelos \& Maia, 2001).

No estudo de Vasconcelos e Maia (2001) encontrou-se uma relação positiva entre a idade e os níveis de prática nas meninas até aos 16 anos e um declínio a partir daí, enquanto nos meninos a partir dos 12 anos até aos 19 houve uma estabilização dos níveis de prática. Os autores apontam como justificação uma maior adesão à AF e desportiva nos tempos livres e na escola, assim como menores hábitos de sedentarismo. No caso do nosso estudo não temos dados que suportem qualquer assunção baseada em fatores externos influenciadores. De qualquer forma sabemos que alguns obstáculos sociais, demográficos, pessoais ou ambientais podem limitar algumas crianças e adolescentes a praticarem AF. As crianças são influenciadas pelos hábitos de AF das pessoas com quem convivem (Landry \& Driscoll, 2012). Desta forma, esta tendência pode estar ligada a alunos que tenham pais que incentivam a sua participação ou que são pais ativos. As circunstâncias familiares, sociais e/ou económicas, estão fortemente relacionadas com o desenvolvimento a educação e oportunidades de prática de AF (Wadsworth, 1999), mostrando uma influência directa, sobretudo dos pais, na AF das crianças (Keresztes, Piko, Pluhar, \& Page, 2008; King, Tergerson, \& Wilson, 2008) ilustrado pelo facto de crianças poderem ser aproximadamente 6 vezes fisicamente mais ativas se tiverem pais ativos (Fuemmeler, Anderson, \& Mâsse, 2011).

Outro fator externo que pode ter influenciado esta tendência é o nível socioeconómico. Não temos dados da condição socioeconómica dos alunos uma vez que o estudo foi de corte transversal. A população em estudo na altura da recolha podia ter um estatuto socioeconómico diferente entre os grupos de idade, sendo os mais novos menos privilegiados que os mais velhos. De facto, o menor nível socioeconómico e educacional tem sido associado com menores níveis de participação em atividades físicas (Sallis \& Owen, 1998).

O facto de ser um estudo realizado num momento só, de corte transversal, pode levar a que tenhamos estudado duas populações com comportamentos diferentes. Não sabemos se o grupo dos mais velhos não manteve os níveis de prática que tinha há um ou dois anos, não sabemos se aumentou, ou se, de acordo com a literatura, diminuiu. Da mesma forma, não sabemos se o grupo que agora pertence aos mais novos vai aumentar os seus níveis de prática quando aumentar a idade, se vai manter, ou se vai diminuir. Podemos estar na presença de um grupo dos mais novos que é mais sedentário que os mais velhos. Não parece ser o caso, uma vez que o IMC, que está negativamente relacionado com a prática da AF (Donnelly et al., 2009), aumentou com a idade $(\mathrm{p}<0.001)$ e não diminuiu. Por exemplo, 
no estudo de Berkey, Rockett, Gillman, e Colditz (2003), o nível de AF foi maior para o grupo dos mais velhos, contudo nesse estudo, essa maior participação estava associada à diminuição da prevalência de sobrepeso com o aumento de idade de $27.3 \%$ aos 10 anos, para $17.3 \%$ aos 15 anos.

Podemos ainda indicar que, pela análise dos intervalos de idade que os sujeitos do grupo dos mais novos estão a terminar o $3^{\circ}$ ciclo do Ensino Básico enquanto os mais velhos estão já em idade de Ensino Secundário. Os alunos do Ensino Secundário têm mais tempo de EF que os alunos do $3^{\circ}$ ciclo. Este facto pode justificar o aumento dos níveis de prática diretamente por terem mais tempo de EF, ou indiretamente pela relação positiva que a prática da $\mathrm{EF}$ poderá ter no nível de prática de AF fora das aulas e fora da escola. Independentemente da qualidade e quantidade de AF nas aulas de EF, Dobbins, Husson, DeCorby, e LaRocca (2013) indicam que crianças que fazem EF têm aproximadamente 3 vezes mais probabilidade de se envolverem em atividades físicas diversas de intensidade moderada a vigorosa durante o seu dia na escola e complementarmente as crianças cujos valores de AF escolares são superiores tendem a ser mais ativas fora da escola e durante os fins-de-semana (Donnelly et al., 2009).

A diminuição da AF com o aumento da idade, apesar de não ter acontecido no nosso estudo, tem sido descrita com frequência (Armstrong \& Welsman, 2006; Armstrong, Welsman, \& Kirby, 2000; Boreham \& Riddoch, 2001; Yli-Piipari et al., 2012). Paralelamente a prevalência da adesão à prática desportiva também é menor à medida que a idade avança. Alves, Silva, Ribeiro, Vermatti, e Fisberg (2000) verificaram que $42.5 \%$ dos adolescentes começaram a desenvolver algum tipo de AF antes dos 12 anos de idade, $51 \%$ entre 12 e 16 anos e $6.5 \%$ após os 16 anos. A interrupção da prática de $\mathrm{AF}$ foi mencionada por $47 \%$ dos adolescentes, ocorrendo após os 15 anos. Pegando na assunção de que uma criança ativa tem maior probabilidade de ser um adulto ativo e verificando que a prevalência é maior em idades mais baixas faz-nos pensar que estamos no bom caminho. Ainda assim os números são muito baixos e a quantidade de crianças e adolescentes que abandonam a prática da AF regular é muito alta. Por exemplo Sallis (1993), numa revisão da especialidade, verificou que as crianças de 6-7 anos realizavam mais $\mathrm{AF}$ de intensidade moderada a vigorosa por dia ( $45 \mathrm{~min} / \mathrm{dia})$ que as crianças com 10 a 16 anos (16-45min/dia). Entre adolescentes com idades compreendidas entre 15 e 18 anos os padrões de AF vão decaindo, sobretudo nas meninas (Boreham \& Riddoch, 2001) e a AF vigorosa regular apresenta um declínio constante entre os 12 até aos 21 anos, estabilizando entre os 30 e 64 anos (Caspersen, Pereira, \& Curran, 2000). Num estudo longitudinal, no sentido de avaliar as alterações aos níveis de AF em crianças com idades dos 11 aos 13 anos, Armstrong et al. (2000) descobriram as seguintes diminuições com a idade de 76.2 para $44.4 \%$ nos meninos e 73.5 para $31.6 \%$ nas meninas. Ainda constataram que com a idade de 11 anos $22.1 \%$ dos meninos e $29.6 \%$ das meninas não realizavam sequer 10 minutos de AF moderada por semana e com a idade de 13 anos os valores foram de 28.4 e $51.9 \%$ para meninos e meninas respetivamente.

A industrialização e o desenvolvimento não são alheios a este abandono e diminuição dos níveis de prática de $\mathrm{AF}$. $\mathrm{O}$ facto de as crianças não poderem brincar na rua ou em locais próprios, por questões de segurança ou outros, leva à instalação de comportamentos sedentários. As evidências apontam no sentido de crianças que passam mais tempo a brincar fora de casa tenderem a ser mais ativos (Hinkley, Crawford, Salmon, Okely, \& Hesketh, 2008).

Relativamente à prática de AF e IMC verificou-se que os alunos normoponderais apresentaram maiores níveis de prática quando comparados com os alunos com sobrepeso ou magros respetivamente $(4.61 \pm 1.73$ vs $4.20 \pm 1.73$ vs 4.06 $\pm 1.77 ; \mathrm{p}=0.044)$. Este resultado, apesar de ser tangencialmente significativo apenas, era esperado e está de acordo com as evidências da literatura que indicam que indivíduos obesos são mais frequentemente hipocinéticos (Wilmore \& Costill, 2004), participando menos em atividades físicas e desportivas (Giugliani \& Carneiro, 2004). 
Relativamente à prevalência de práticas desportivas federadas, encontramos valores de prática de $71.8 \%$ nos meninos e $58.8 \%$ nas meninas, sem efeito nem da idade, nem do IMC. Este dado indica que o facto de o aluno ter sobrepeso ou obesidade não o inibe de praticar AF fora da escola. Curiosamente mais meninos praticam AF desportiva, durante mais tempo (3.73 vs 2.69 horas por semana para meninos e meninas respetivamente), mas a prevalência de excesso de peso foi maior nos meninos ( $p>0.05)$. Contudo alguns fatores mais, além dos que já foram referidos, podem estar na origem desta menor participação em atividades físicas. Por exemplo: a menor participação dos alunos com sobrepeso pode dever-se às complicações ortopédicas que normalmente estas populações apresentam (Fisberg, 1995), contudo num trabalho de revisão recente, Shultz, Anner, e Hills (2009) não indicaram ter encontrado correlações entre as dores nas articulações ou desconforto em algumas tarefas com a diminuição da AF em crianças obesas; ou por outro lado, o facto de ter excesso de peso ou ser obeso, juntamente com o processo de aumento de peso, carrega consigo consequências sociais, económicas e psicológicas (Keller \& Stevens, 1996).

\section{IMC}

No que diz respeito ao IMC a nossa amostra apresentou valores de IMC entre 12.99 e 38.48 $\mathrm{kg} / \mathrm{m}^{2}$ e uma média de $20.54 \pm 3.48 \mathrm{~kg} / \mathrm{m}^{2}$. Estes dados isoladamente não nos dão muita informação, contudo, relativamente às prevalências a nossa amostra apresentou uma percentagem de indivíduos com sobrepeso de $25.3 \%$, normoponderais de $71.4 \%$ e magros de $3.3 \%$.

A comparação dos dados serve para normalizar os nossos resultados, contudo o rácio de $1 \mathrm{em}$ cada 4 criança ou adolescente ter sobrepeso é preocupante, sendo $27.8 \%$ meninos e $22.9 \%$ meninas. Os nossos valores de maior prevalência nos meninos estão de acordo com os resultados de Albuquerque, Nóbrega, Samouda, e Manco (2012) e divergem dos resultados de Silva et al. (2008), ambos em crianças portuguesas. Contudo, estudos noutras populações vêm indicando há alguns anos a mesma tendência de prevalên- cia, maior nos meninos que nas meninas (Maffeis, Schutz, Piccoli, Gonfiantini, \& Pinelli, 1993), assim como meninas com valores de IMC superiores ao dos meninos (Jung, 1997).

Os dados disponíveis da população portuguesa indicam-nos valores de prevalência diversos. Por exemplo, Padez, Mourão, Moreira, e Rosado (2005) apresentaram valores de $31,3 \%$ de crianças com sobrepeso; Silva et al. (2008) apresentaram valores de $16.3 \%$ de crianças com sobrepeso; Rito et al. (2012) apresentaram valores de $28.1 \%$ de sobrepeso; e Albuquerque et al. (2012) apresentaram valores de sobrepeso de $33 \%$ indicando que esta prevalência aumentou $40 \%$ nos últimos 10 anos.

Relativamente à população adolescente, o estudo de Ferreira e Duarte (2013) indicou uma prevalência de sobrepeso de $23.5 \%$ para os meninos e $21.4 \%$ para as meninas, sendo os valores de obesidade respetivamente de $5.4 \%$ e $3.4 \%$.

Os dados da população portuguesa aproximam-nos dos valores dos países do sul da Europa (Albuquerque et al., 2012) o que, juntamente aos valores de inatividade física mostrados por Armstrong e Welsman (2006) onde os mesmos países aparecem na cauda dos 22 países estudados, levanta muitas preocupações. De facto este aumento de prevalência tem vindo a aumentar na população em geral e cada vez aparece mais cedo em idades mais baixas (Carreira, Pereira, Azevedo, \& Lunet, 2012).

Os dados são preocupantes e devem ser vistos com cautela. Os trabalhos referidos anteriormente utilizaram todos os pontos de corte do International Obesity Task Force, ou seja, o IMC. Antunes e Moreira (2011), relativamente à população portuguesa e comparando vários padrões numa revisão sistemática, indicaram os seguintes valores relativos ao IMC de $8.6 \%$ a $29.6 \%$ em masculinos e de $8.8 \%$ a $31.4 \%$ em femininos. O que estes resultados querem dizer é que independentemente do método utilizado, o IMC varia dentro da mesma população ou país de acordo com vários fatores sociodemográficos (MarquesVidal \& Dias, 2005). A nossa amostra foi recrutada de várias escolas do litoral de Portugal, fazendo com que as comparações sejam difíceis de fazer. 
Relativamente à magreza, os dados do WHO (2013) indicam valores em crianças com menos de 5 anos de $1.5 \%$ para a população europeia, sendo que este valor diminuiu desde a última avaliação quase 8 vezes, deixando patente a tendência para o gradual aumento do peso globalmente. Rito et al. (2012) reportou valores de $4.8 \%$ de magreza, idêntico ao reportado por Marques-Vidal, Ferreira, Oliveira, e Paccaud (2008) 4.7 para meninas e $5.3 \%$ para meninos.

Sardinha et al. (2011) indicaram uma prevalência de sobrepeso e obesidade menor à medida que a idade avança. No nosso estudo o valor de IMC médio aumentou o que poderá dever-se ao próprio crescimento ou aumento da massa muscular ou devido aos efeitos cumulativos do estado do IMC das idades anteriores (Gortmaker, Dietz, Sobol, \& Wehler, 1987).

Um aspeto que pode ser determinante na prevalência da obesidade dos alunos é o estatuto socioeconómico (Cardoso \& Padez, 2008). Num estudo realizado por Nogueira et al. (2013), as crianças com um estatuto socioeconómico mais baixo tinham mais probabilidade de serem obesas e de participarem menos nas atividades físicas e desportivas. Este aspeto também não foi contemplado na recolha dos nossos dados.

\section{Perceção de Sucesso Escolar}

A maior parte dos alunos do nosso estudo define o sucesso escolar através da aprovação com boas classificações (81.8\%) sendo este valor maior nos alunos mais novos, nos alunos com peso normal e nas meninas. Os alunos cuja perceção face ao sucesso escolar era passar de ano com boas notas reportaram níveis superiores de prática $(\mathrm{p}=0.03)$ que os restantes grupos. Além disso apresentaram também melhor média final e classificação final a Português, Matemática e EF. Estes resultados parecem indicar uma relação entre a prática de AF e desportiva e o sucesso escolar. De facto, a AF é essencial para um desenvolvimento saudável e a inatividade física, cada vez mais normal entre as crianças, cria condições para constrangimentos não só ao nível da saúde e capacidades físicas mas também ao nível cognitivo (Booth, Gordon, Carlson, \& Hamilton, 2000). Por exemplo, Ahamed et al. (2007), num estudo randomizado, realizaram intervenção em várias escolas no sentido de aumentar a AF relacionando a mesma com o desempenho escolar. Não encontraram diferenças entre meninos e meninas no desempenho escolar e apesar do grupo de controlo ter começado o estudo com melhores classificações que o grupo experimental, no final das 16 semanas de AF acrescida não foi encontrada qualquer diferença no desempenho escolar. No nosso estudo as meninas têm melhores classificações na média final e a todas as disciplinas com exceção de EF que é inferior à dos meninos.

O nosso estudo apresentou relações positivas entre a classificação da EF com Português ( $r=$ .36; $\mathrm{p}<0.001)$ e com Matemática $(\mathrm{r}=.42 ; \mathrm{p}<$ $0.001)$. Estes resultados convergem com os de Chomitz et al. (2009), que realizaram um estudo tentando relacionar as capacidades em matemática e língua e os resultados em testes de aptidão física realizados na EF. Os resultados caminharam paralelamente mostrando que a probabilidade do aluno ter melhores classificações a qualquer uma das áreas estava diretamente relacionada com o número de testes de aptidão física concluídos com sucesso. Contudo não foi possível descobrir a direção desta relação. São melhores alunos porque são mais aptos fisicamente ou o contrário? No nosso estudo a prática de $\mathrm{AF}$ apresentou uma relação positiva com a classificação em EF, não se verificando diferenças nas classificações de EF entre meninos e meninas, e se considerarmos a prática de atividades desportivas federadas encontrámos uma relação positiva com a média final e com a EF. Na realidade, a AF pode influenciar a saúde e capacidade de pensar em crianças, levando a melhorias do desempenho escolar e a um cérebro mais eficiente durante mais tempo (Hillman, Kamijo, \& Scudder, 2011). Estudos transversais têm mostrado associações positivas entre a AF e a aptidão cardiovascular no sucesso escolar (Tomporowski, Davis, Miller, \& Naglieri, 2007; Trudeau \& Shephard, 2008). O nosso estudo, apesar de não ter avaliado a aptidão cardiovascular, encontrou uma relação positiva entre as classificações de matemática e português e a classificação de EF $(p<0.001)$. A esta relação pode-se aduzir que há uma relação entre a EF e a 
prática de AF. Os alunos que reportaram uma atitude mais positiva face ao sucesso escolar, que era passar com boas notas, apresentaram relação positiva com a prática de AF. A atitude face ao sucesso por sua vez teve relação com o rendimento escolar média final, matemática e português.

Uma análise mais aprofundada em estudos com outro tipo de desenho tem mostrado resultados inconsistentes (Ahamed et al., 2007; Carlson et al., 2008; Coe, Pivarnik, Womack, Reeves, \& Malina, 2006; Sibley \& Etnier, 2003). Contudo os resultados devem ser vistos com algum cuidado, já que diferenças nos desenhos de estudo, no tempo de intervenção, nos métodos e na população podem resultar em más interpretações. O estudo de Donnelly et al. (2009) foi realizado através de um desenho de investigação longitudinal, procurando relações entre a AF e o desempenho escolar, ao contrário dos estudos de Castelli, Hillman, Buck, e Erwin (2007) e de Coe et al. (2006) que basearam os seus resultados em relações entre sucesso escolar e testes de aptidão física.

Os benefícios a nível cognitivo podem ter paralelismo com os da aptidão física nas suas diferentes componentes, que aumentam quando o exercício é realizado com a devida intensidade e regularidade, mas que também diminuem com períodos de inatividade. O estudo de Davis et al. (2007) indica que 20 minutos de AF aeróbia por dia (a uma intensidade média de $150 \mathrm{bpm}$ ) é insuficiente para se obterem ganhos nos processos cognitivos das crianças (foram realizados testes cognitivos em controlo, 20 minutos dia e $40 \mathrm{mi}$ nutos dia). As diferenças foram encontradas nos 40 minutos dia indicando a existência de um limiar a partir do qual há um efeito dose-resposta (Davis et al., 2007). Talvez o maior benefício do aumento da AF seja a sua relação com as capacidades cognitivas e o desempenho escolar. Por exemplo, no estudo de Trudeau e Shephard (2008) a AF adicional dentro da aula de EF manteve os resultados escolares ou aumentou os mesmos, apesar da redução do tempo em sala de aula. Da mesma forma Donnelly et al. (2013) verificaram que o desempenho escolar melhorou com o aumento da AF escolar.
Num estudo longitudinal, Carlson et al. (2008) acompanharam os alunos durante todo o ensino primário registando a quantidade de tempo em aulas de EF e o desempenho escolar em matemática e língua, concluindo que a EF não teve efeitos adversos no desempenho escolar e que limitar a EF não só não é desejável como pode ter o efeito oposto. De facto, o mecanismo de associação entre a AF e o desempenho escolar envolve capacidade de concentração, memória, processamento da informação e comportamento (Trudeau \& Shephard, 2008).

Pode haver uma relação entre o efeito agudo da AF na concentração dos alunos, mas o efeito crónico da AF vigorosa com o desempenho escolar não está ainda bem reforçada pela ciência (Taras, 2005), não só na aptidão cardiorrespiratória como na aptidão muscular. No sentido de encontrar relações entre capacidades físicas específicas e o desempenho escolar em matemática e língua, Eveland-Sayers, Farley, Fuller, Morgan e Caputo (2009) encontraram uma relação positiva com a matemática nos testes de aptidão muscular $(\mathrm{r}=$ .20). No estudo de Castelli et al. (2007), os testes de aptidão física relacionaram-se positivamente com o desempenho escolar geral, em matemática e línguas, sobretudo a capacidade aeróbia, enquanto o IMC apresentou uma relação negativa. De facto não basta ter um IMC normal. A AF regular e sobretudo os níveis de aptidão física podem ser mais importantes. Num estudo longitudinal entre o $4^{\circ}$ e $9^{\circ}$ ano, comparando aqueles que são fisicamente aptos com aqueles que são menos ativos, foram encontradas diferenças nos resultados de matemática e línguas, sendo estas diferenças maiores para as meninas. Os autores concluíram que a aptidão física geral é um melhor preditor do desempenho escolar do que o IMC (London \& Castrechini, 2011).

O nosso estudo verificou um efeito significativo do IMC na média final $\left(\eta^{2}=0.02\right)$ e na classificação de EF $\left(\eta^{2}=0.09\right)$ mostrando que os alunos com peso normal tiveram melhores classificações que os alunos com sobrepeso $(p<0.05)$. De facto, o sobrepeso em crianças tem sido relacionado com menor desempenho escolar (Dwyer, Sallis, Blizzard, Lazarus, \& Dean, 2006; Taras \& Potts-Datema, 2005). Outro fator que pode ajudar na magnitude deste efeito é também a menor 
participação dos alunos com sobrepeso na prática de AF. Os alunos participam menos e as suas classificações são mais baixas.

Além dos problemas associados já referenciados, o sobrepeso e obesidade em crianças estão inversamente associados com resultados em testes de Inteligência (QI) e desempenho escolar. Exemplo disso são os estudos de: Campos, Sigulem, Moraes, Escrivão e Fisberg (1996), onde crianças obesas obtiveram resultados inferiores nos testes de QI do que crianças com peso normal com diferenças nos testes de QI (85 vs 91, respetivamente), além de que as últimas demonstraram um leque de interesses mais vasto que as crianças obesas, melhor capacidade de socialização e maior destreza; o estudo de Li (1995) onde crianças com obesidade severa obtiveram resultados inferiores no teste de QI que crianças com peso normal; e a revisão realizada por Taras e PottsDatema (2005) onde reforçaram as evidências atualizadas da relação inversa do sobrepeso e obesidade com o desempenho escolar.

Outra explicação possível para estes resultados inferiores face às populações com peso normal, pode ter a ver com a função executiva, que pode estar comprometida no indivíduo com sobrepeso devido à falta de AF. A função executiva diz respeito ao desenvolvimento e aplicação de estratégias, auto regulação, intencionalidade, e utilização do conhecimento e desenvolve-se desde a infância até a adolescência (Best, 2010). A este propósito, Davis et al. (2007) indicaram uma relação direta entre AF vigorosa regular e a função executiva em crianças, aduzindo que 40 minutos por dia de AF aeróbia vigorosa resultam numa maior capacidade de habilidades de organização entre crianças obesas. Este aumento da capacidade da função executiva pode permitir a estas crianças aprender mais rapidamente a curto prazo.

Apesar de haver apenas um efeito positivo na média final e na classificação de EF, a tendência global de todas as classificações é ser menor neste grupo do que no grupo normoponderal. Além disso, na atitude face ao sucesso escolar, apesar da prevalência entre os indivíduos com sobrepeso ter sido passar de ano com boas notas, temos quase $20 \%$ do grupo que indica que basta passar de ano independentemente de ter ou não boas notas $(\mathrm{p}<0.01)$. Uma vez que a AF aumenta a socialização, promove a função executiva e reduz a depressão, parece razoável considerar que a mesma possa trazer benefícios ao desempenho escolar (Landry \& Driscoll, 2012).

Vista de outra forma, é possível não dizer que a prática de $\mathrm{AF}$ está diretamente relacionada com o sucesso escolar, mas é possível confirmar que a mesma tem impacto positivo no combate à obesidade e que esta tem impacto positivo no sucesso escolar (Donnelly et al., 2013).

\section{CONCLUSÕES}

De acordo com os objetivos enunciados e das análises realizadas observamos que os meninos apresentaram níveis de prática de atividade física e atividade desportiva federada superiores às meninas havendo um aumento da prevalência dos níveis de atividade física com o aumento da idade. Verificou-se também que os alunos com sobrepeso apresentaram níveis inferiores de prática de atividade física quando comparados com os alunos com peso normal ou magros.

Relativamente ao IMC, a amostra estudada apresentou uma prevalência de sobrepeso de $25.3 \%$, maior no sexo masculino $(27.8 \%)$ que no feminino (22.9\%), aumentando esta com a idade.

Relativamente à perceção de sucesso escolar $\mathrm{e}$ fatores influenciadores, a maior parte dos alunos (81.8\%) considerou que o sucesso escolar é passar com boas notas, destacando-se uma maior prevalência de meninas com esta perceção face ao sucesso escolar. Neste contexto verificou-se que os alunos com esta atitude face ao sucesso escolar revelaram maiores níveis de prática de atividade física havendo maior prevalência de alunos com peso normal com esta perceção face ao sucesso escolar.

Relativamente ao rendimento escolar verificamos que o rendimento a EF está relacionado positivamente com o rendimento a língua portuguesa e matemática, assim como a níveis superiores de prática de atividade física. A prática de desporto federado tem relação positiva com o rendimento escolar média final e EF em todo o grupo e relação positiva com o rendimento escolar a língua portuguesa nas meninas. Verificamos 
também um efeito do IMC no rendimento escolar na média final e em EF, onde os alunos com sobrepeso têm um rendimento escolar inferior.

Face aos resultados consideramos a prática de atividade física, além de catalisador de níveis de IMC mais baixos, um fator com influência positiva no rendimento escolar em alunos dos ensinos básico e secundário.

\section{Agradecimentos: \\ Nada a declarar}

Conflito de Interesses:

Nada a declarar.

Financiamento:

Nada a declarar.

\section{REFERÊNCIAS}

Ahamed, Y., Macdonald, H., Reed, K., Naylor, P.-J., Liu-Ambrose, T., \& McKay, H. (2007). Schoolbased physical activity does not compromise children's academic performance. Medicine and Science in Sports and Exercise, 39(2), 371-376. http://doi.org/10.1249/01.mss.0000241654.455 $00.8 \mathrm{e}$

Albuquerque, D., Nóbrega, C., Samouda, H., \& Manco, L. (2012). Assessment of obesity and abdominal obesity among Portuguese children. Acta Médica Portuguesa, 25(3), 169-173.

Alves, S., Silva, S., Ribeiro, R., Vermatti, A., \& Fisberg, M. (2000). Avaliaçäo de atividade física, estado nutricional e condiçäo social em adolescentes. Folha médica, 119(1), 26-33.

Antunes, A., \& Moreira, P. (2011). [Prevalence of overweight and obesity in Portuguese children and adolescents]. Acta médica portuguesa, 24(2), 279-284.

Armstrong, N. (1995). Children's cardiopulmonary fitness and physical activity patterns: the European scene. Em C. J. R. Blimkie \& O. Bar-Or (Eds.), New Horizons in Pediatric Exercise Science (pp. 177-189). Champaign, IL: Human Kinetics.

Armstrong, N., \& Van Mechelen, W. (1998). Are children fit and active? Em S. Biddle, J. F. Sallis, \& N. Cavill (Eds.), Young and active? Young people and health-enhancing physical activity-evidence and implications (pp. 69-97). London: Health Education Authority.
Armstrong, N., \& Welsman, J. R. (2006). The physical activity patterns of European youth with reference to methods of assessment. Sports Medicine (Auckland, N.Z.), 36(12), 1067-1086.

Armstrong, N., Welsman, J. R., \& Kirby, B. J. (2000). Longitudinal changes in 11-13-year-olds' physical activity. Acta Paediatrica (Oslo, Norway: 1992), 89(7), 775-780.

Azevedo Junior, M. R., Araújo, C. L. P., \& Pereira, F. M. (2006). Atividades físicas e esportivas na adolescência: mudanças de preferências ao longo das últimas décadas. Revista Brasileira de Educação Física e Esporte, 20(1), 51-58.

Berkey, C. S., Rockett, H. R. H., Gillman, M. W., \& Colditz, G. A. (2003). One-year changes in activity and in inactivity among 10- to 15-year-old boys and girls: relationship to change in body mass index. Pediatrics, 111(4 Pt 1), 836-843.

Best, J. R. (2010). Effects of Physical Activity on Children's Executive Function: Contributions of Experimental Research on Aerobic Exercise. Developmental Review: DR, 30(4), 331-551.

Blair, S. N., LaMonte, M. J., \& Nichaman, M. Z. (2004). The evolution of physical activity recommendations: how much is enough? The American Journal of Clinical Nutrition, 79(5), 913S-920S.

Booth, F. W., Gordon, S. E., Carlson, C. J., \& Hamilton, M. T. (2000). Waging war on modern chronic diseases: primary prevention through exercise biology. Journal of Applied Physiology (Bethesda, Md.: 1985), 88(2), 774-787.

Boreham, C., \& Riddoch, C. (2001). The physical activity, fitness and health of children. Journal of Sports Sciences, 19(12), 915-929. http://doi.org/10.1080/026404101317108426

Campos, A. L., Sigulem, D. M., Moraes, D. E., Escrivão, A. M., \& Fisberg, M. (1996). [Intelligent quotient of obese children and adolescents by the Weschler scale]. Revista de saúde pública, 30(1), 85-90.

Cardoso, H. F. V., \& Padez, C. (2008). Changes in height, weight, BMI and in the prevalence of obesity among 9- to 11-year-old affluent Portuguese schoolboys, between 1960 and 2000. Annals of Human Biology, 35(6), 624-638. http://doi.org/10.1080/03014460802464200

Carlson, S. A., Fulton, J. E., Lee, S. M., Maynard, L. M., Brown, D. R., Kohl, H. W., 3rd, \& Dietz, W. H. (2008). Physical education and academic achievement in elementary school: data from the early childhood longitudinal study. American Journal of Public Health, 98(4), 721-727. http://doi.org/10.2105/AJPH.2007.117176

Carreira, H., Pereira, M., Azevedo, A., \& Lunet, N. (2012). Trends of BMI and prevalence of overweight and obesity in Portugal (1995-2005): a systematic review. Public Health Nutrition, 15(6), 972-981. http://doi.org/10.1017/S1368980012000559

Caspersen, C. J., Pereira, M. A., \& Curran, K. M. (2000). Changes in physical activity patterns in the United States, by sex and cross-sectional age. 
Medicine and science in sports and exercise, 32(9), 1601-1609.

Castelli, D. M., Hillman, C. H., Buck, S. M., \& Erwin, H. E. (2007). Physical fitness and academic achievement in third- and fifth-grade students. Journal of Sport \& Exercise Psychology, 29(2), 239-252.

Chomitz, V. R., Slining, M. M., McGowan, R. J., Mitchell, S. E., Dawson, G. F., \& Hacker, K. A. (2009). Is there a relationship between physical fitness and academic achievement? Positive results from public school children in the northeastern United States. The Journal of School Health, 79(1), 3037. 1561.2008.00371.x http://doi.org/10.1111/j.1746-

Cliff, D. P., Okely, A. D., Smith, L. M., \& McKeen, K. (2009). Relationships between fundamental movement skills and objectively measured physical activity in preschool children. Pediatric Exercise Science, 21(4), 436-449.

Cloes, M. (2004). Motivation in the Gym: A Qualitative Approach. Em Global Perspectives in the Integration of Physical Activity, Sports, Dance, and Exercise Science in Physical Education: From Theory to Practice (pp. 267-280). Hong Kong: Hong Kong Institute of Education.

Cloes, M., Laraki, N., Dubuisson, J., \& Theunissen, C. (2007). Attitudes, perception of physical education's objectives and self-competence in secondary school students. Comparison of according to their BMI. Em The role of physical education and sport in promoting physical activity and health (pp. 68-78). Jyväskylä: University of Jyväskylä, Department of Sport Sciences Research.

Coe, D. P., Pivarnik, J. M., Womack, C. J., Reeves, M. J., \& Malina, R. M. (2006). Effect of physical education and activity levels on academic achievement in children. Medicine and Science in Sports and Exercise, 38(8), 1515-1519. http://doi.org/10.1249/01.mss.0000227537.131 $75.1 \mathrm{~b}$

Cohen, J. (1988). Statistical Power Analysis for the behavioral sciences (2. ${ }^{\mathrm{a}}$ ed.). Hillsdale, NJ: Lawrence Erlbaum Associates.

Cole, T. J., Bellizzi, M. C., Flegal, K. M., \& Dietz, W. H. (2000). Establishing a standard definition for child overweight and obesity worldwide: international survey. BMJ, 320(7244), 1240. http://doi.org/10.1136/bmj.320.7244.1240

Cole, T. J., Flegal, K. M., Nicholls, D., \& Jackson, A. A. (2007). Body mass index cut offs to define thinness in children and adolescents: international survey. BMJ (Clinical research ed.), 335(7612), 194.

http://doi.org/10.1136/bmj.39238.399444.55

Cole, T. J., \& Lobstein, T. (2012). Extended international (IOTF) body mass index cut-offs for thinness, overweight and obesity. Pediatric obesity, 7(4), 284-294. http://doi.org/10.1111/j.20476310.2012.00064.x
Corbin, C. B. (2002). Physical Activity for Everyone: What Every Physical Educator Should Know about Promoting Lifelong Physical Activity. Journal of Teaching in Physical Education, 21(2), $128-44$.

Davis, C. L., Tomporowski, P. D., Boyle, C. A., Waller, J. L., Miller, P. H., Naglieri, J. A., \& Gregoski, M. (2007). Effects of Aerobic Exercise on Overweight Children's Cognitive Functioning. Research quarterly for exercise and sport, 78(5), 510-519.

Dobbins, M., Husson, H., DeCorby, K., \& LaRocca, R. L. (2013). School-based physical activity programs for promoting physical activity and fitness in children and adolescents aged 6 to 18. Em Cochrane Database of Systematic Reviews (The Cochrane Library, Vol. 2). New Jersey: John Wiley \& Sons, Ltd. Obtido de http://onlinelibrary.wiley.com/doi/10.1002/14651858.CD007 651.pub2/abstract

Donnelly, J. E., Greene, J. L., Gibson, C. A., Smith, B. K., Washburn, R. A., Sullivan, D. K., ... Williams, S. L. (2009). Physical Activity Across the Curriculum (PAAC): a randomized controlled trial to promote physical activity and diminish overweight and obesity in elementary school children. Preventive Medicine, 49(4), 336-341. http://doi.org/10.1016/j.ypmed.2009.07.022

Donnelly, J. E., Greene, J. L., Gibson, C. A., Sullivan, D. K., Hansen, D. M., Hillman, C. H., ... Washburn, R. A. (2013). Physical activity and academic achievement across the curriculum (A + PAAC): rationale and design of a 3 -year, cluster-randomized trial. BMC Public Health, 13, 307. http://doi.org/10.1186/1471-2458-13-307

Dwyer, T., Sallis, J. F., Blizzard, L., Lazarus, R., \& Dean, K. (2006). Relation of Academic Performance to Physical Activity and Fitness in Children. Pediatr Exerc Sci, 13(3), 225 - 237.

Eckel, R. H., Grundy, S. M., \& Zimmet, P. Z. (2005). The metabolic syndrome. Lancet, 365(9468), 1415-1428. http://doi.org/10.1016/S01406736(05)66378-7

Eveland-Sayers, B. M., Farley, R. S., Fuller, D. K., Morgan, D. W., \& Caputo, J. L. (2009). Physical fitness and academic achievement in elementary school children. Journal of Physical Activity \& Health, 6(1), 99-104.

Ferreira, F. S., \& Duarte, J. A. (2013). Overweight, obesity, physical activity, cardiorespiratory and muscular fitness in a Portuguese sample of high school adolescents. Minerva Pediatrica, 65(1), 83-91.

Fisberg, M. (1995). Obesidade na infância e na adolescência. São Paulo: Fundo Editorial Byk.

Freedman, D. S., Khan, L. K., Dietz, W. H., Srinivasan, S. R., \& Berenson, G. S. (2001). Relationship of childhood obesity to coronary heart disease risk factors in adulthood: the Bogalusa Heart Study. Pediatrics, 108(3), 712-718. 
Fuemmeler, B. F., Anderson, C. B., \& Mâsse, L. C. (2011). Parent-child relationship of directly measured physical activity. The International Journal of Behavioral Nutrition and Physical Activity, 8, 17. http://doi.org/10.1186/1479-58688-17

Giugliani, R., \& Carneiro, E. C. (2004). Fatores associados à obesidade em escolares. Jornal de Pediatria, 80(1), 17-22.

Gonçalves, H., Hallal, P. C., Amorim, T. C., Araújo, C. L. P., \& Menezes, A. M. B. (2007). [Sociocultural factors and physical activity level in early adolescence]. Revista Panamericana De Salud Pública = Pan American Journal of Public Health, 22(4), 246-253.

Gortmaker, S. L., Dietz, W. H., Jr, Sobol, A. M., \& Wehler, C. A. (1987). Increasing pediatric obesity in the United States. American Journal of Diseases of Children (1960), 141(5), 535-540.

Guerra, S., Santos, P., Ribeiro, J., Duarte, J., \& Mota, J. (2003). Assessment of children's and adolescent's physical activity levels. Eur Phys Educ Rev, 9, 75-85.

Heath, G. W., Pratt, M., Warren, C. W., \& Kann, L. (1994). Physical activity patterns in American high school students. Results from the 1990 Youth Risk Behavior Survey. Archives of Pediatrics \& Adolescent Medicine, 148(11), 11311136.

Hillman, C. H., Kamijo, K., \& Scudder, M. (2011). A review of chronic and acute physical activity participation on neuroelectric measures of brain health and cognition during childhood. Preventive Medicine, 52 Suppl 1, S21-28. http://doi.org/10.1016/j.ypmed.2011.01.024

Hinkley, T., Crawford, D., Salmon, J., Okely, A. D., \& Hesketh, K. (2008). Preschool children and physical activity: a review of correlates. American Journal of Preventive Medicine, 34(5), 435-441. http://doi.org/10.1016/j.amepre.2008.02.001

Jaakkola, T., Kalaja, S., Liukkonen, J., Jutila, A., Virtanen, P., \& Watt, A. (2009). Relations among physical activity patterns, lifestyle activities, and fundamental movement skills for Finnish students in grade 7. Perceptual and Motor Skills, 108(1), 97-111.

Jung, R. T. (1997). Obesity as a disease. British Medical Bulletin, 53(2), 307-321.

Kelder, S. H., Mitchell, P. D., McKenzie, T. L., Derby, C., Strikmiller, P. K., Luepker, R. V., \& Stone, E. J. (2003). Long-term implementation of the CATCH physical education program. Health Education \& Behavior: The Official Publication of the Society for Public Health Education, 30(4), 463-475.

Keller, C., \& Stevens, K. R. (1996). Assessment, etiology, and intervention in obesity in children. The Nurse Practitioner, 21(9), 31-36, 38, 41-42.

Keresztes, N., Piko, B. F., Pluhar, Z. F., \& Page, R. M. (2008). Social influences in sports activity among adolescents. The Journal of the Royal Society for the Promotion of Health, 128(1), 21-25.
King, K. A., Tergerson, J. L., \& Wilson, B. R. (2008). Effect of social support on adolescents' perceptions of and engagement in physical activity. Journal of Physical Activity \& Health, 5(3), 374384.

Landry, B. W., \& Driscoll, S. W. (2012). Physical activity in children and adolescents. $P M \& R$ : The Journal of Injury, Function, and Rehabilitation, $4(11)$, $826-832$. http://doi.org/10.1016/j.pmrj.2012.09.585

Lee, I. M., Paffenbarger, R. S., Jr, \& Hennekens, C. H. (1997). Physical activity, physical fitness and longevity. Aging (Milan, Italy), 9(1-2), 2-11.

Li, X. (1995). A study of intelligence and personality in children with simple obesity. International Journal of Obesity and Related Metabolic Disorders: Journal of the International Association for the Study of Obesity, 19(5), 355-357.

London, R. A., \& Castrechini, S. (2011). A longitudinal examination of the link between youth physical fitness and academic achievement. The Journal of School Health, 81(7), 400-408. http://doi.org/10.1111/j.17461561.2011.00608.x

Maffeis, C., \& Castellani, M. (2007). Physical activity: an effective way to control weight in children? Nutrition, Metabolism, and Cardiovascular Diseases: $\quad N M C D, \quad 17(5), \quad 394-408$. http://doi.org/10.1016/j.numecd.2006.08.006

Maffeis, C., Schutz, Y., Piccoli, R., Gonfiantini, E., \& Pinelli, L. (1993). Prevalence of obesity in children in north-east Italy. International Journal of Obesity and Related Metabolic Disorders: Journal of the International Association for the Study of Obesity, 17(5), 287-294.

Marques-Vidal, P., \& Dias, C. M. (2005). Trends in overweight and obesity in Portugal: the National Health Surveys 1995-6 and 1998-9. Obesity Research, 13(7), 1141-1145. http://doi.org/10.1038/oby.2005.135

Marques-Vidal, P., Ferreira, R., Oliveira, J. M., \& Paccaud, F. (2008). Is thinness more prevalent than obesity in Portuguese adolescents? Clinical Nutrition (Edinburgh, Scotland), 27(4), 531-536. http://doi.org/10.1016/j.clnu.2008.04.002

Martínez-González, M. A., Martínez, J. A., Hu, F. B., Gibney, M. J., \& Kearney, J. (1999). Physical inactivity, sedentary lifestyle and obesity in the European Union. International Journal of Obesity and Related Metabolic Disorders, 23(11), 11921201.

Mateus, A., Dias, C., Corte-Real, N., Garganta, J., \& Fonseca, A. (2010). Percepção de competência física no futebol feminino e masculino: um estudo com jovens atletas e seus pais. Revista Iberoamericana de Psicología del Ejercicio y el Deporte, 5(1), 61-78.

McArdle, W. D., Katch, F. I., \& Katch, V. L. (2010). Exercise Physiology: Nutrition, Energy, and Human Performance. Lippincott Williams \& Wilkins. 
McKenzie, T. L., Marshall, S. J., Sallis, J. F., \& Conway, T. L. (2000). Student activity levels, lesson context, and teacher behavior during middle school physical education. Research Quarterly for Exercise and Sport, 71(3), 249-259.

Mota, J., \& Sallis, J. F. (2002). Actividade Física e Saúde. Factores de Influência da Actividade Física nas Crianças e nos Adolescentes. Campo das Letras.

Mota, J., Santos, P., Guerra, S., Ribeiro, J., \& Duarte, J. (2002). Differences of daily physical activity levels of children according to body mass index. Pediatr Exerc Sci, 14, 442-52.

NES. (2002). FitnessGram ® Manual de aplicação de testes (Núcleo de Exercício e Saúde da Faculdade de Motricidade Humana). Lisboa: Impriluz.

Nogueira, H., Gama, A., Mourão, I., Marques, V., Ferrão, M., \& Padez, C. (2013). The associations of SES, obesity, sport activity, and perceived neighborhood environments: is there a model of environmental injustice penalizing Portuguese children? American Journal of Human Biology: The Official Journal of the Human Biology Council, 25(3), 434-436. http://doi.org/10.1002/ajhb.22384

Oehlschlaeger, M. H. K., Pinheiro, R. T., Horta, B., Gelatti, C., \& San'Tana, P. (2004). [Prevalence of sedentarism and its associated factors among urban adolescents]. Revista de saúde pública, 38(2), 157-163.

Ogden, C. L., Carroll, M. D., Curtin, L. R., McDowell, M. A., Tabak, C. J., \& Flegal, K. M. (2006). Prevalence of overweight and obesity in the United States, 1999-2004. JAMA: The Journal of the American Medical Association, 295(13), 15491555. http://doi.org/10.1001/jama.295.13.1549

Okely, A. D., Booth, M. L., \& Patterson, J. W. (2001). Relationship of physical activity to fundamental movement skills among adolescents. Medicine and Science in Sports and Exercise, 33(11), 1899-1904.

Padez, C., Mourão, I., Moreira, P., \& Rosado, V. (2005). Prevalence and risk factors for overweight and obesity in Portuguese children. Acta Paediatrica (Oslo, Norway: 1992), 94(11), 15501557.

http://doi.org/10.1080/08035250510042924

Prochaska, J. J., Sallis, J. F., \& Long, B. (2001). A physical activity screening measure for use with adolescents in primary care. Archives of Pediatrics \& Adolescent Medicine, 155(5), 554-559.

Rasberry, C. N., Lee, S. M., Robin, L., Laris, B. A., Russell, L. A., Coyle, K. K., \& Nihiser, A. J. (2011). The association between school-based physical activity, including physical education, and academic performance: a systematic review of the literature. Preventive Medicine, 52 Suppl 1, S1020. http://doi.org/10.1016/j.ypmed.2011.01.027

Rito, A., Wijnhoven, T. M. A., Rutter, H., Carvalho, M. A., Paixão, E., Ramos, C., ... Breda, J. (2012). Prevalence of obesity among Portuguese children
(6-8 years old) using three definition criteria: COSI Portugal, 2008. Pediatric Obesity, 7(6), 413-422. http://doi.org/10.1111/j.20476310.2012.00068.x

Sallis, J. F. (1993). Epidemiology of physical activity and fitness in children and adolescents. Critical Reviews in Food Science and Nutrition, 33(4-5), 403-408. http://doi.org/10.1080/10408399309527639

Sallis, J. F., McKenzie, T. L., Beets, M. W., Beighle, A., Erwin, H., \& Lee, S. (2012). Physical education's role in public health: steps forward and backward over 20 years and HOPE for the future. Research Quarterly for Exercise and Sport, 83(2), 125-135.

Sallis, J. F., \& Owen, N. (1998). Physical Activity and Behavioral Medicine. SAGE Publications.

Santos, P., Guerra, S., Ribeiro, J. C., Duarte, J. A., \& Mota, J. (2003). Age and gender-related physical activity. A descriptive study in children using accelerometry. The Journal of Sports Medicine and Physical Fitness, 43(1), 85-89.

Sardinha, L. B., Santos, R., Vale, S., Silva, A. M., Ferreira, J. P., Raimundo, A. M., ... Mota, J. (2011). Prevalence of overweight and obesity among Portuguese youth: a study in a representative sample of 10-18-year-old children and adolescents. International Journal of Pediatric Obesity: IJPO: An Official Journal of the International Association for the Study of Obesity, 6(2-2), e124-128. http://doi.org/10.3109/17477166.2010.490263

Shephard, R. J., \& Trudeau, F. (2000). The legacy of physical education: Influences on adult lifestyle. Pediatric Exercise Science, 12(1), 34-50.

Shultz, S. P., Anner, J., \& Hills, A. P. (2009). Paediatric obesity, physical activity and the musculoskeletal system. Obesity Reviews: An Official Journal of the International Association for the Study of Obesity, 10(5), 576-582. http://doi.org/10.1111/j.1467789X.2009.00587.x

Sibley, B. A., \& Etnier, J. L. (2003). The relationship between physical activity and cognition in children: a meta-analysis. Pediatric Exercice Science, 15(3), 243-256.

Silva, A. J., Mourão-Carvalhal, M. I., Reis, V. M., Mota, M. P., Garrido, N. D., Pitanga, F., \& Marinho, D. (2008). The prevalence of overweight and obesity among portuguese children. Fitness \& Performance Journal, 7(5), 301-305.

Taras, H. (2005). Physical activity and student performance at school. The Journal of School Health, 75(6), 214-218. http://doi.org/10.1111/j.17461561.2005.00026.x

Taras, H., \& Potts-Datema, W. (2005). Obesity and student performance at school. The Journal of School Health, 75(8), 291-295. http://doi.org/10.1111/j.17461561.2005.00040.x

Taylor, W. C., Baranowski, T., \& Sallis, J. F. (1994). Family determinants of childhood physical activity: a social-cognitive model. Em R. K. Dishman 
(Ed.), Advances in exercise adherence (pp. 319342). Champaign, IL: Human Kinetics.

Tomporowski, P. D., Davis, C. L., Miller, P. H., \& Naglieri, J. A. (2007). Exercise and Children's Intelligence, Cognition, and Academic Achievement. Educational Psychology Review, 20(2), 111-131. http://doi.org/10.1007/s10648-007-9057-0

Trost, S. G., Sallis, J. F., Pate, R. R., Freedson, P. S., Taylor, W. C., \& Dowda, M. (2003). Evaluating a model of parental influence on youth physical activity. American journal of preventive medicine, 25(4), 277-282.

Trudeau, F., \& Shephard, R. J. (2008). Physical education, school physical activity, school sports and academic performance. The International Journal of Behavioral Nutrition and Physical Activity, 5, 10. http://doi.org/10.1186/1479-5868-5-10

US Department of Health and Human Services. (1996). Physical activity and health: a report of the Surgeon General. Atlanta, Georgia: US DHHS, Centers for Disease Control and Prevention, National Center for Chronic Disease Prevention and Health Promotion. Obtido de http://www.cdc.gov/nccdphp/sgr/sgr.htm
Vasconcelos, M., \& Maia, J. (2001). Actividade física de crianças e jovens - haverá um declínio? Estudo transversal em indivíduos dos dois sexos dos 10 aos 19 anos de idade. Revista Portuguesa de Ciências do Desporto, 1(3), 44-52.

Wadsworth, M. E. J. (1999). Early life. Em M. Marmot \& R. Wilkinson (Eds.), Social Determinants of Health (pp. 44-58). Oxford: Oxford University Press.

Weinberg, R. S., \& Gould, D. (1997). Psychologie du sport et de l'activité physique. Edisem.

WHO. (2013). World Health Statistics 2013.

WHO/CDC. (2005). Physical Activity Strategy: An Action Plan for Promotion/Implementation. World Health Organization. Obtido de http://www.who.int/moveforhealth/publications/mfh_miami_planaction_feb05.pdf

Wilmore, J. H., \& Costill, D. L. (2004). Physiology of Sport and Exercise. Human Kinetics Publishers.

Yli-Piipari, S., Leskinen, E., Jaakkola, T., \& Liukkonen, J. (2012). Predictive role of physical education motivation: the developmental trajectories of physical activity during grades 7-9. Research Quarterly for Exercise and Sport, 83(4), 560-569.

Todo o conteúdo da revista Motricidade está licenciado sob a Creative Commons, exceto quando especificado em contrário e nos conteúdos retirados de outras fontes bibliográficas. 